\title{
Cost-Effectiveness of Primary Care
}

\author{
Richard A. Deyo, MD, MPH
}

The resident describes a new patient in clinic today, a 40-year-old woman with a cbolesterol reading of $240 \mathrm{mg} / \mathrm{dL}$, but no other coronary risk factors. She asks whether it would be cost-effective to begin therapy with "statin" drugs to lower the patient's cholesterol. The attending physician, always skeptical of bigh technology and eager to promote preventive care, says "Sure it is. Some of the statin drugs now cost about $\$ 50$ a month. Compare that with $\$ 50,000$ for a coronary bypass later on if she isn't treated now."

This article is intended only as an introduction to the use of cost-effectiveness analysis in primary care. The goals are to provide a clear understanding of the difference between the cost of a treatment and its cost-effectiveness; consider what is generally a socially acceptable range for cost-effectiveness; provide some basic criteria for critically evaluating cost-effectiveness analyses in the medical literature; give some examples of the cost-effectiveness of various treatments in primary care; and provide for comparison some examples of costeffectiveness in the world of specialty care. For those interested in more detail, excellent books and reviews are available, including the report of a US. Public Health Service-appointed expert panel. ${ }^{1}$

Cost-effective care is that judged to provide good health value for expenditure. Health value refers to the benefits of a particular medical intervention, which might include longer life, better quality of life, or both. Expenditures should include not only the costs of a test or treatment itself, but the subsequent costs it might cause, including additional medical interventions, work disability, costs of long-term care, and so forth.

Cost-effective does not necessarily mean cheap. The attending physician described above has made a common mistake - equating cost with cost-effectiveness, and assuming that low-tech care is more

Submitted, revised 29 October 1998.

From the Department of Medicine, and the Department of Health Services and Center for Cost and Outcomes Research, University of Washington, Seattle. Address reprint requests to Richard A. Deyo, MD, MPH, Department of Health Services and Center for Cost and Outcomes Research, Box 358853, University of Washington, Seattle, WA 98195-8853.

This project was supported in part by grant number HS-08194 from the Agency for Health Care Policy and Research. cost-effective than high-tech care. Cost-effectiveness is always a ratio between cost and effectiveness, so some cheap interventions might not be costeffective. No matter how inexpensive it is, if the effectiveness of an intervention is low, the costeffectiveness will be poor. On the other hand, expensive interventions do not necessarily have poor cost-effectiveness. If they also happen to be effective, then the cost to effectiveness ratio might be favorable. Cost-effective does not necessarily mean cost saving; at a logical extreme, no care at all would be cost saving, but not cost-effective. Some introductory examples can serve to illustrate these points.

We might all agree that a guaiac card for fecal occult blood is an inexpensive test. In a study published 20 years ago, the authors estimated the cost of guaiac cards to be $\$ 4$ for the first test and $\$ 1$ for each subsequent test. At the time, performing 6 stool guaiacs was the norm for the regular screening test. Neuhauser and Lweicki ${ }^{2}$ undertook a costeffectiveness analysis to determine whether performing all six screening tests was a reasonable strategy. Their analysis assumed that for the first guaiac card some cancers will be found, and with this inexpensive test the cost per case of cancer detected proved to be about $\$ 1200$. With the second guaiac card there is less cancer left to be detected. The effectiveness of the second guaiac card is therefore a little bit lower than the first, even though the price is also lower. With the third guaiac card some additional cases of cancer are still detected, but now the yield is even lower. Most malignancies have been picked up with the first two cards, and not many more are detected with the third guaiac. The cost of the third stool guaiac was estimated to be $\$ 49,000$ per additional case of can- 
cer detected (in 1975 dollars). By the time of the sixth guaiac card, even though it costs $\$ 1$ for that guaiac card, the cost-effectiveness is about $\$ 47$ million for each additional case of cancer detected. Why so dismal? Because not much additional cancer is detected after those first five guaiac cards have already been used. So we have a cheap test, but the cost per case detected is enormous, even at $\$ 1$ per card. This example illustrates the importance of examining the incremental value of additional expenditures.

On the other hand, consider coronary artery bypass surgery. Here we have an intervention that costs perhaps $\$ 30,000$ per operation. At least for the most high-risk patients, who gain the most in terms of life-expectancy, the cost per year of life saved can be quite good. Even though it is an expensive intervention, if we consider left main coronary bypass vs medical management, we have a cost-effectiveness ratio of about $\$ 2300$ to $\$ 5600$ per year of life saved, a ratio that most people would find quite acceptable. ${ }^{3}$

If we compare three-vessel disease with left main coronary disease, then the cost-effectiveness of bypass surgery is not quite as good $(\$ 12,000$ per year of life saved) but still in a generally acceptable range. This finding illustrates that it is important to consider exactly to whom we offer the intervention under consideration. If we look at two-vessel disease, a still milder form, the cost-effectiveness is somewhat worse again, not because the operation is more expensive, but because it does not have as big a "bang for the buck" in terms of lives saved or years of life added. The cost-effectiveness now is in the range of $\$ 28,000$ to $\$ 75,000$ per year of life saved, ${ }^{3}$ and we are getting close to a threshold at which many people begin to ask, "Is it worth it?"4

\section{What is Acceptable Cost-Effectiveness?}

There are no hard-and-fast rules about what level of cost-effectiveness is acceptable, and any suggestions in this regard will be debated. As a rough guide, however, we as a society generally accept treatments as appropriate if they cost less than about $\$ 50,000$ for a quality-adjusted life-year gained (a conclusion from Laupacis et al, ${ }^{4}$ but roughly updated and converted from Canadian to US dollars). Such treatments are almost always accepted as part of our routine clinical repertoire, and we do many every day. For interventions in the range of $\$ 50,000$ to $\$ 120,000$ per quality-adjusted $\$$ life-year (to be defined later), we generally begin to say that this cost is high, and it is not so clear ino some cases that the bang is worth the buck. We often provide services in that range, but access iș sometimes limited. Interventions that cost moret than $\$ 120,000$ per quality-adjusted life-year gained? are often challenged and are infrequently imple $-\overrightarrow{\vec{*}}$ mented on a large scale. ${ }^{4}$ These are not rules but describe how we behave about cost-effectiveness, for better or worse.

\section{When is Cost-Effectiveness Analysis Important?}

Cost-effectiveness analysis does not need to be ap plied to everything that we do. In some situations, it makes no sense to bother with a formal analysis? One example occurs when a new test or a treatment is both cheaper and more effective (or even equallyi effective) than the older standard intervention, al-으 though some analysis is necessary to determine that ${ }_{c}^{-}$ this case is true. Also, there is usually little point in doing a cost-effectiveness analysis if we have not 월 shown the effectiveness of a treatment, because this effectiveness is part of the cost-effectiveness ratio. $\varnothing$ If we have a treatment of unknown effectiveness, then we cannot know its cost-effectiveness. In somes circumstances, we might perform a cost-effectiveness analysis and decide that treatment effective-迹 ness would have to be implausibly great to make the $\overrightarrow{\vec{A}}$ therapy cost-effective, and it is therefore not worth 3 pursuing the intervention at all. But by and large, when we have no clue about the effectiveness of treatment or a test, we should wait before attempting the cost-effectiveness analysis. Thus, we gener-产. ally want to do cost-effectiveness analysis when we $\exists$ have a new test or a new treatment that is botho응 more expensive and more effective than the old 0 treatments that were available. If a test or treatment N is both less expensive and less effective, we might $>$ also ask whether the saving is worth the loss or whatever we are giving up in health benefits.

\section{How Do We Describe the Effectiveness Part of $\frac{\circ}{\bar{C}}$ the Ratio?}

One problem in cost-effectiveness analysis is quan- $\frac{T}{T}$ tifying effectiveness, providing a number to be used $\frac{\text { }}{\mathbb{1}}$ in the cost-effectiveness ratio. One conceptually $\frac{?}{2}$ simple measure of effectiveness is years of life saved, something about which we all understand 8 
and have some shared agreement. The notion of quality-adjusted life-years is more complex. Most of us would agree that there are differences in quality of life. We do not want to give the same credit to a life-saving treatment that leaves somebody blind for the next 10 years as we do to a treatment that leaves perfect vision for the next 10 years. Those treatments are not equally effective, so we penalize the treatment that reduces quality of life and give it less credit for effectiveness. How to make these quality adjustments remains highly controversial. How do we quantify the value of being able to see? Techniques for approaching this quantification are described in detail elsewhere. ${ }^{1}$

One might think about effectiveness in terms of diagnoses made for a diagnostic test. For example, we examined the cost per case of cancer detected for the stool guaiac test. Some analyses examine cost per hospitalization prevented or some other problem prevented. Of course, not all hospital days prevented or cancers found are the same in terms of health impact, introducing ambiguity even for these measures.

But many benefits of treatment are hard to measure and are poorly accounted for in these kinds of analyses. An example is the use of sonography during pregnancy. For some patients, there is an emotional benefit to sonography, a reassurance value, and the thrill of seeing a sonogram of the baby. Some women say they are willing to pay for this benefit. But such effects typically are hard to account for in cost-effectiveness analyses, because they have little bearing on years of life or more than momentary quality of life.

\section{How Do We Measure the Cost Part of the Ratio?}

The costs of a medical service seem deceptively easy to measure. The charge for direct medical care is the easiest to capture. It might be difficult to actually figure out the total care costs for an illness, however, and we often substitute charges for costs. Unfortunately, what we charge for a service is not the same as the real resource cost, the real value of that service. Also, there are other costs that we all recognize as important and that might be affected by a treatment. An example is the cost of home care for a frail elderly person who cannot care for himself. Someone bears that cost, and if it is not a family member, we must pay as a society for someone to provide that service. The cost of work absenteeism or reduced productivity is another exam-
Table 1. Critically Evaluating Published CostEffectiveness Analyses.

1. Is the question clear? The treatment and the targeted patient population should be precisely described, and the viewpoint (societal, payer, provider) should be specified.

2. Is the alternative strategy clearly defined along with its costs and benefits? The analysis should represent incremental costs and effectiveness beyond the alternative strategy.

3. Has the effectiveness of the intervention been convincingly demonstrated?

4. Are future costs and benefits discounted compared with current costs and benefits?

5. Was a sensitivity analysis performed, examining the effects of varying key values (costs or effectiveness) through a plausible range?

6. Are there other cost-effectiveness analyses available on the same topic? Are the results concordant?

Adapted from Drummond et al. ${ }^{5}$

ple. This cost is real; somebody pays for it. For most purposes cost-effectiveness analysts recommend this broad societal perspective in performing cost-effectiveness analyses. If costs to the family or costs to the employer are substantially improved by a treatment, they might offset the direct medical cost, but it is more difficult to get a handle on the value of such nonmedical services.

Finally, economists are concerned about the opportunity costs of providing tests and treatments. This term refers to the fact that if we spend our money doing one thing, we cannot spend it for doing something else. If we spend an extra $\$ 500$ million doing coronary bypass surgery, there is $\$ 500$ million less for prenatal care, cancer screening, or other services, unless we agree to raise the overall cost (insurance premiums, taxes) of medical care. Thus, the clinical decisions we make become important for resource allocation and can influence other day-to-day clinical decisions.

\section{Critically Evaluating Cost-Effectiveness Analyses}

Published cost-effectiveness studies vary widely in quality, and clinicians should become critical readers. ${ }^{5}$ Table 1 lists some helpful criteria in evaluating published studies. First, it is important that the question be crystal clear. What patients are we talking about? As we have seen, the cost-effectiveness of a single treatment can vary widely with the types of patients to whom the treatment is applied. All patients with coronary disease, for example, are not the same. Exactly what is the intervention be- 
ing studied? Home-based renal dialysis has a costeffectiveness very different from hospital-based renal dialysis, for example. What is the viewpoint being considered? Is it the societal perspective on cost (after including work loss, out-of-pocket costs, and contributions of time by the family), the direct medical perspective on costs (just the costs of medical care), or an even narrower view, the insurance company's perspective on costs (what they will actually reimburse). An analyst might take any of those viewpoints.

Second, it is important to describe the competing therapy, because there is almost always some alternative treatment for the condition being studied. We must account for the cost of that, too, because if we are not doing the new treatment, we are still doing the old treatment, with its particular costs and effectiveness. The cost-effectiveness of a new intervention must therefore be compared with some standard intervention, which must be clearly described. We want to know that the analysis represents the incremental cost-effectiveness beyond the normal or usual alternative treatment rather than no treatment at all (unless that is the usual approach).

The third point was made earlier, that we must understand the effectiveness of the treatment or the test before we can meaningfully study its costeffectiveness. Fourth, the authors should select all the relevant and important costs that one might consider and discount future costs or savings. In a formal cost-effectiveness analysis it is important to consider future costs and future outcomes - but also important to discount those somewhat. Discounting is a way of acknowledging that receiving $\$ 100$ now is preferred to receiving $\$ 10010$ years from now. Why? You might not be alive 10 years from now to enjoy that $\$ 100$. Furthermore, if you get the $\$ 100$ now and invest it, 10 years from now you will have more than a $\$ 100$. So saving costs now is also more valuable than saving equivalent future costs. Many analysts would say medical benefits are the same. If a treatment restores good sight now and for the next 10 years, the benefit is much more valuable than a treatment that restores sight 10 years from now. One would rather not put in 10 years of blindness to get the benefit. So it might be important to discount outcomes as well as the costs, and there are techniques for doing so. ${ }^{1}$

Next is the concept of sensitivity analysis. The idea here is that most cost-effectiveness analysts must make educated guesses about certain $\operatorname{costs}_{2}^{c}$ aspects of effectiveness, and time frames. Guess work is necessary because all the details about treat-o ment costs, the costs of complications, future sav- 은 ings, indirect costs, effectiveness in specific patients subgroups, and other important data are rarelyo available in definitive form. So an analyst might ask, What about a worst case scenario? What if the costs really are worse than I think, and what if the effectiveness really is less than I think? Alterna $-\frac{\overline{0}}{\bar{\sigma}}$ tively, what is the best case scenario if the cost is actually lower than I think, and the effectiveness is actually better than I think? Repeating the analysiss with different values for key variables is called $a_{\omega}$ sensitivity analysis, and one can vary many factors in this approach, singly or simultaneously.

Finally, it is nice to know when we read cost $-\frac{\overrightarrow{3}}{3}$ effectiveness studies whether there are other cost $-\overrightarrow{\bar{\omega}}$ effectiveness analyses on the same topic for comparison. Comparison is important, because such analyses sometimes reach discordant conclusions. Cost-effectiveness analysis is not a highly precise science; there is a fair bit of art involved, and investigators come up with different answers. Sometimes they are sufficiently different to make a substantial difference in clinical decisions. On the other hand, concordant results from multiple analyses suggest a robust finding.

\section{Examples from Primary Care} You are a member of the formulary committee for your $\stackrel{\overrightarrow{3}}{\overrightarrow{3}}$ bealth maintenance organization, which decides whether? to add and pay for new drugs as part of the bealth plan. Today's agenda includes a discussion of nicotine gum and nicotine patches, as well as misoprostol for preventing.

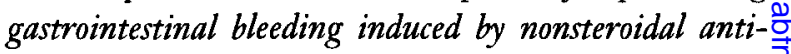
inflammatory drugs. The chief of pulmonary medicine makes an impassioned plea for adding nicotine gum to응 the formulary, while the bead of rbeumatology wants to 9 be sure misoprostol is added. The pharmacy director $\sim$ \% opposes both, saying they would be too expensive for the plan. You ask what the cost-effectiveness of these treatments is, get only shrugs, and bead off to the library after ${ }_{\omega}^{N}$ the meeting.

The precise methodology will not be belabored $\stackrel{\circ}{\varnothing}$ here, but for purposes of comparability, the exam- $\stackrel{D}{\rightarrow}$ ples discussed here include only direct medical 0

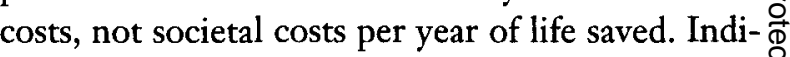
rect costs are not considered, nor is quality adjust- $\frac{\overrightarrow{0}}{\otimes}$ ment for years of life. Thus, these are the simplest but perhaps the least controversial types of analy- 8 


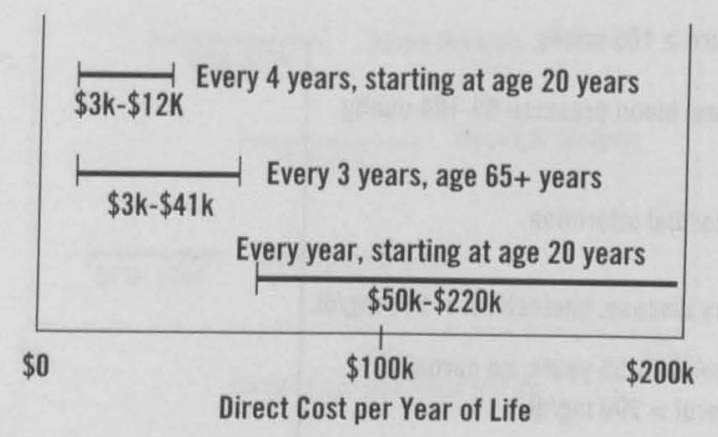

Figure 1. Example: cervical cancer screening.

ses. The studies have been adjusted to show data in 1993 dollars, by previous analysts. ${ }^{3}$ These treatments are theoretically life-saving, and so the costeffectiveness is expressed as cost per year of life saved. Although one might object to using these terms for the analyses, they at least offer illustrative examples for which costs and effectiveness are expressed in the same metric. The figures show graphs, called league tables by economists, comparing the cost-effectiveness of different interventions. These graphic comparisons make some economists and policy makers uncomfortable, because they can imply greater precision of these measures than is justified, and the direct comparisons might be taken too literally. Such comparisons seem essential, however, if cost-effectiveness analysis is to have any practical value for resource allocation decisions.

Figure 1 displays published values for the costeffectiveness of cervical cancer screening and makes several important points. First, cost-effectiveness is expressed in ranges, reflecting various published values rather than a single number. For many interventions there is a fairly substantial range of published values. Second, the importance of patient targeting and precise definition of the intervention are illustrated here. Cancer screening for young women every 4 years has better cost-effectiveness than cancer screening every year. Screening every 4 years reduces the cost and retains most of the effectiveness. Cost-effectiveness is a little worse for people 65 years and older because the benefits are somewhat less. On the other hand, cost-effectiveness of cervical cancer screening every year starting at the age of 20 years looks like a relatively poor value. This strategy adds costs but provides only modest added benefit in terms of additional cancer detected, resulting in a range of cost-effectiveness estimates for which many would raise eyebrows and ask whether we can really afford it on a large scale.

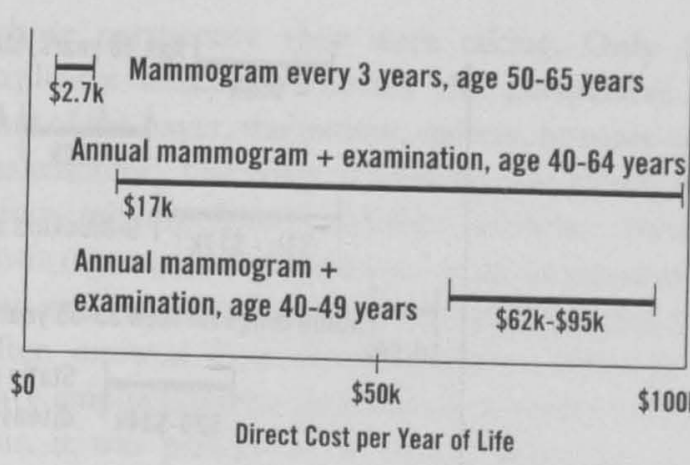

Figure 2. Example: breast cancer screening.

Figure 2 shows data for breast cancer screenin Again, there is a range of published values, depend ing in part on which populations are considere $\vec{N}$ Mammography every 3 years for women aged 50 है 65 years enjoys well-demonstrated efficacy, an cost-effectiveness is good. For annual mammogr $\vec{\omega}$ phy and examinations starting at a younger agi cost-effectiveness estimates vary widely froig $\$ 17,000$ per year of life saved to $\$ 100,000$ per yeart of life saved. If one considers annual mammogr phy and breast examination for only women age 40 to 49 years, effectiveness is lower because thern are fewer cases of cancer to detect, and cost-effeco tiveness starts at a more daunting range.

Preventive care for cervical and breast cance relies on early detection, a form of secondary pre vention. What about primary prevention strate gies? Preventing influenza, for example, is ver cost-effective. Some recent cost-effectiveness anal yses suggest that for certain patient groups influ릉 enza prevention might actually be cost saving, cost ing less to perform flu vaccinations than not tos perform them when taking into account all the costs of influenza and its complications. The esti-产 mate in Figure 3 is less than $\$ 500$ per year of life saved, also very favorable.

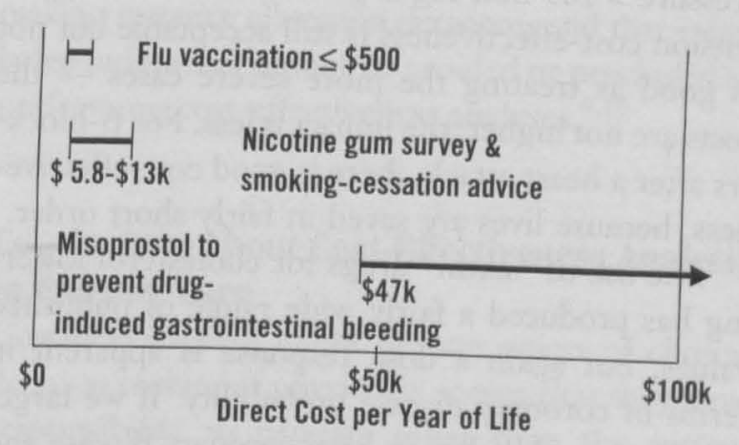

Figure 3. Some preventive treatments. 


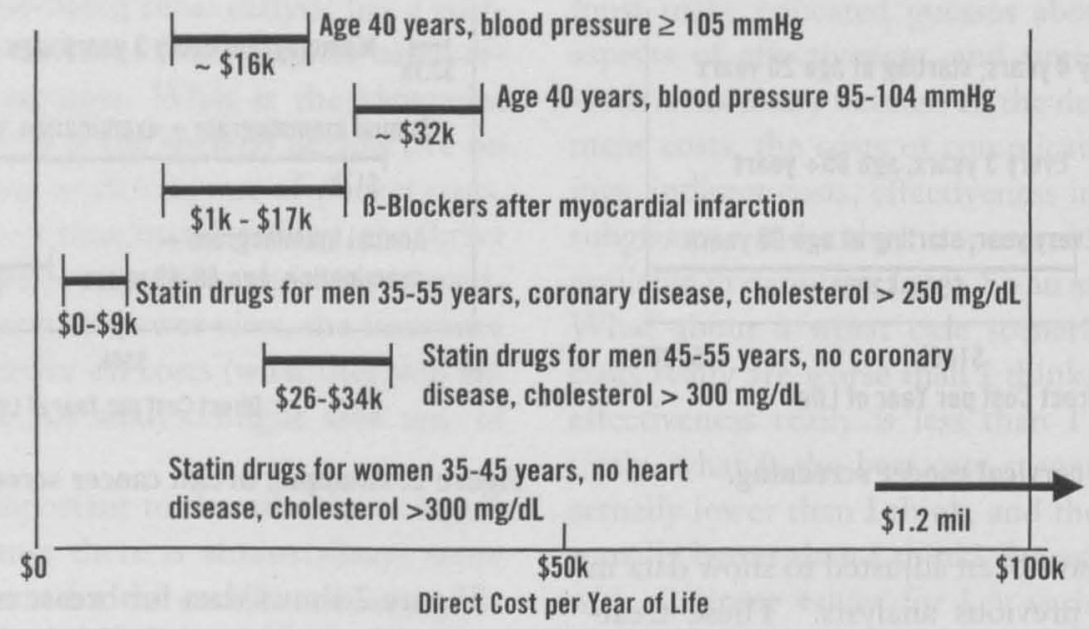

Figure 4. Examples: hypertension and $\beta$-blockers after myocardial infarction.

Returning to the scenario that began this section, our member of the formulary committee discovers that nicotine gum and smoking cessation advice are also quite cost-effective (Figure 3). These interventions are in the favorable range of $\$ 6000$ to $\$ 12,000$ per year of life saved. Contrast that with misoprostol to prevent drug-induced gastrointestinal bleeding. This intervention is expensive per year of life saved, in part because saving a life with this type of treatment is rare. We might prevent gastrointestinal bleeding, but that does not necessarily mean a life saved or a year of life saved, because most bleeding episodes are not life-threatening. Estimates range up to $\$ 200,000$ per year of life saved. Our formulary committee member returns to the next meeting with a well-articulated argument in favor of adding nicotine gum, but not misoprostol.

Figure 4 illustrates the cost-effectiveness of some cardiovascular disease interventions. The cost per year of life saved for prescribing medications for patients aged 40 years with a diastolic blood pressure $>105 \mathrm{~mm} \mathrm{Hg}$ is good. For milder hypertension cost-effectiveness is still acceptable but not as good as treating the more severe cases - the costs are not higher, the impact is less. For $\beta$-blockers after a heart attack, there is good cost-effectiveness, because lives are saved in fairly short order.

The use of "statin" drugs for cholesterol lowering has produced a fairly wide range of published values, but again a dose response is apparent in terms of coronary disease probability. If we target young men who already have coronary disease and high cholesterol levels, cost-effectiveness is good.
For middle-aged men with no coronary disease, even with a high cholesterol level cost-effectiveness is not quite so good. For a still lower risk group, young women with no heart disease, even with high cholesterol levels, we might be in the range of a \$1 million per year of life saved. This unattractive situation was presented in the scenario that began this article.

\section{Examples of Public Health Interventions}

For contrast, we can consider some public health interventions that have little to do with the medical world. ${ }^{3}$ As suggested in Figure 5, mandating automatic vs manual seat belts in cars has a favorable cost-effectiveness somewhere between $\$ 0$ and $\$ 25,000$ per year of life saved. Federal laws requiring smoke detectors in the home and chlorination of drinking water also appear highly cost-effective. Banning asbestos in automobile brake blocks gets up to $\$ 30,000$ to $\$ 40,000$ per year of life saved; perhaps worth doing, but more expensive. Banning

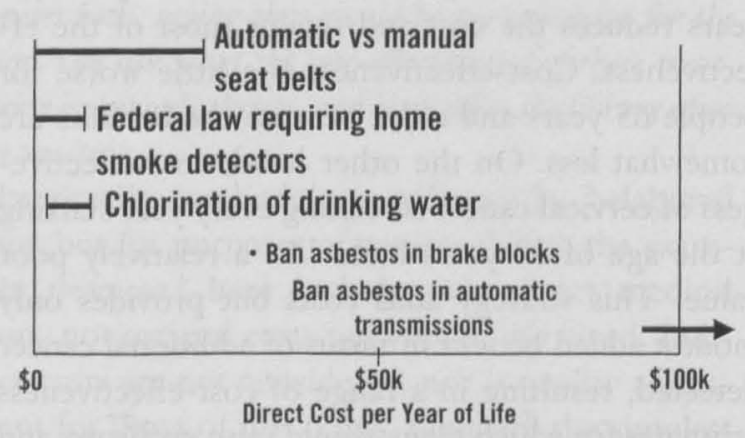

Figure 5. Some public health and safety interventions. 


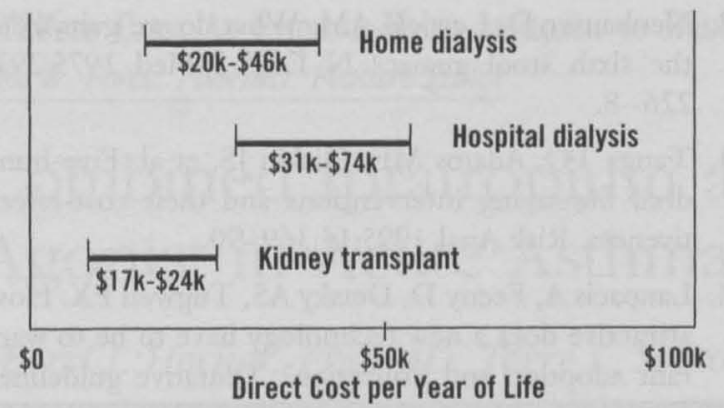

Figure 6. Specialty care: treatment for end-stage renal disease.

asbestos in automatic transmissions is out in the range of millions of dollars per year of life saved. This public health intervention might not be worth the cost.

\section{Examples from Specialty Care}

At the opposite extreme from public health, we might consider highly specialized forms of medical care. $^{3}$ We examined the example of surgery for coronary artery disease, and Figure 6 shows some examples from nephrology for home dialysis, hospital dialysis, and kidney transplantation. Although kidney transplantation can be expensive, it is also effective. Thus, its cost-effectiveness is in a reasonable range. Home dialysis is more expensive per life year saved, and hospital dialysis is more expensive still. This illustrates the importance of precisely defining treatments, because the cost-effectiveness of all dialysis is not the same.

\section{Common Flaws in Publisbed Studies}

The published literature on cost-effectiveness analysis has many flaws, emphasizing the need for a critical eye. For example, Table 2 lists some problems found in 46 published cost-effectiveness analyses. ${ }^{6}$ Most studies did not explicitly describe

Table 2. Common Problems in Cost-Effectiveness Analyses (46 articles, 1985-1987).

\begin{tabular}{lc}
\hline & Percent \\
\hline Perspective explicitly stated & 13 \\
Costs of side effects included & 35 \\
Induced costs included & 4 \\
Averted costs included & 24 \\
Sensitivity analysis done & 35 \\
\hline
\end{tabular}

Reprinted, with permission from Udvarhelyi et al. ${ }^{6}$ whose perspective they were taking. Only 13\% explicitly indicated whether the perspective was that of the payer, the patient, society, or some other stakeholder. The costs of treating side effects were often missing from published analyses. Induced costs (eg, necessary additional tests or monitoring) and averted costs (eg, costs of complications) were often omitted from these analyses. Although we have emphasized the importance of sensitivity analysis, it was performed in only a minority of the studies reviewed. Thus, many published studies are seriously flawed, and the quick critical appraisal is important. A detailed list of criteria for performing and reporting cost-effectiveness analyses was recently published in a prominent medical journal. ${ }^{7}$

An important problem that is often ignored in cost-effectiveness analyses is the opportunity cost of providing certain treatments, even if they appear to be relatively cost-effective. Opportunity cost gets to the problem of making good resource-allocation decisions, and cost-effectiveness analysis is only one component of such decision making. As an example, a recent cost-effectiveness analysis showed that the additional costs of tissue plasminogen activator as opposed to streptokinase for thrombolysis when treating acute myocardial infarction was relatively cost-effective. ${ }^{8}$ The incremental cost-effectiveness beyond that of streptokinase was $\$ 33,000$ per life-year saved and would increase the survival rate of acute myocardial infarction by approximately $1.1 \%$. If this strategy were implemented nationally, the additional cost of health care overall would be $\$ 500$ million. Where would this money come from? Would it come from other medical treatments that would be made unavailable? Would a reluctant public be willing to increase their taxes or their insurance premiums to reduce the risk of death by $1.1 \%$ after a myocardial infarction? This problem illustrates that cost-effectiveness analysis is not the only relevant factor in making resource allocation decisions, and that valueladen judgments cannot be avoided or precluded by performing cost-effectiveness analyses. ${ }^{9,10}$

\section{Conclusions About Cost-Effectiveness Analysis in Primary Care}

Cost-effectiveness might be one aspect of clinical decision making if physicians accept that they have responsibility to patients other than the patient facing them in the examining room. Many would 
argue, however, that the role of cost-effectiveness in individual patient care is modest, and that simple treatment effectiveness should be the first priority. ${ }^{11}$ Determining effective treatment is the first step in practicing evidence-based medicine, and cost-effectiveness simply adds a refinement to this consideration. Clinicians are likely to find costeffectiveness analyses useful, however, in their common roles in helping to set clinical policies. Many physicians are involved in developing drug formularies, clinical guidelines, or decisions about allocating resources within an organization. All these roles for the physician can benefit from including cost-effectiveness analysis as one component of the decision making. Furthermore, for physician executives, cost-effectiveness could often be an important component of decision making.

In summary, cost and cost-effectiveness are quite different. We should insist on good evidence of effectiveness before accepting speculative estimates of cost-effectiveness. It is important to remember that low-tech interventions are not necessarily cost-effective, nor are high-tech interventions necessarily characterized by poor cost-effectiveness. Finally, it is important to remember that patient targeting is critical, and that applying interventions where they offer the most benefit is a key to making the most of the cost-effectiveness of our care.

Pamela J. Hillman helped prepare this article, and Drs. Scott D. Ramsey, Sean D. Sullivan, and Hanan S. Bell contributed to many useful conversations.

\section{References}

1. Gold ME, Siegel JE, Russell LB, Weinstein MC, editors. Cost-effectiveness in health and medicine. New York: Oxford University Press, 1996.
2. Neuhauser D, Lewicki AM. What do we gain from the sixth stool guaiac? N Engl J Med 1975;293:س 226-8.

3. Tengs TO, Adams ME, Pliskin JS, et al. Five-hun-ד dred life-saving interventions and their cost-effec $\stackrel{9}{3}$ tiveness. Risk Anal 1995;15:369-90.

4. Laupacis A, Feeny D, Detsky AS, Tugwell PX. How? ? attractive does a new technology have to be to war $-\frac{\vec{F}}{\bar{\omega}}$ rant adoption and utilization? Tentative guidelineso for using clinical and economic evaluations. CMAJ들 1992;146:473-81.

5. Drummond MF, Richardson WS, O'Brien BJ, Le- $\stackrel{\overparen{D}}{\circ}$ vine $M$, Heyland D. Users' guides to the medical ${ }^{\infty}$ literature. XIII. How to use an article on economic. analysis of clinical practice. A. Are the results of the study valid? Evidence-Based Medicine Working Group. JAMA 1997;277:1552-7.

6. Udvarhelyi IS, Colditz GA, Rai A, Epstein AM.? Cost-effectiveness and cost-benefit analyses in the ${ }_{-}^{\omega}$ medical literature. Are the methods being used cor- $A$ rectly? Ann Intern Med 1992;116:238-44.

7. Siegel JE, Weinstein MC, Russell LB, Gold MR. $\vec{c}$ Recommendations for reporting cost-effectiveness analyses. Panel on Cost-Effectiveness in Health and Medicine. JAMA 1996;276:1339-41.

8. Mark DB, Hlatky MA, Califf RM, et al Cost effec-ঃ tiveness of thrombolytic therapy with tissue plasminogen activator as compared with streptokinase for acute myocardial infarction. N Engl J Med 1995;332: 긍 1418-24.

9. Singer PA. Resource allocation: beyond evidence- $\stackrel{\mathbb{D}}{=}$ based medicine and cost-effectiveness analysis. ACPO J Club 1997;127:A16-8.

10. Gafni A. Economic evaluation of health care interventions: an economist's perspective. ACP J Club 1996;124:A12-4.

11. Detsky AS, Naglie IG. A clinician's guide to costeffectiveness analysis. Ann Intern Med 1990; 113:147-54. 